\title{
Building Assessment Web Service from Question Type Learning Objects
}

\author{
Vytautas Reklaitis \\ Graduate School of Information Systems, \\ University of Electro-Communications, Tokyo, Japan \\ vytas@is.uec.ac.jp \\ Kazys Baniulis Nerijus Aukstakalnis \\ Computer Networking Department, Kaunas University of Technology \\ Kaunas, Lithuania \\ kazys.baniulis@ktu.lt \\ nerijus.aukstakalnis@ktu.It
}

\begin{abstract}
In this paper we discuss the TestTool system as an established testing system model, the one that is being used in real educational settings and supports self-assessment as well as testing learning practices. We then elaborate how this learning object-based system is being re-engineered and extended within the context of Web service oriented architecture. Testing Web service implementation along with considerations regarding how e-learning services from distributed Learning Objects could be composed is given in the final part of the paper.
\end{abstract}

Keywords: assessment, testing, e-learning, Web services.

\section{INTRODUCTION}

The computational Grid technology moved toward Web Services Resource Framework [1] and is becoming available to e-learning community. The success in exploitation of the technology depends on the development of both the Grid infrastructure and the e-learning applications appropriate to this infrastructure. There is a great interest in using Web service oriented technology in e-learning [2, 3, 4, 5], but due to its novelty there is a significant gap between technological Grid advancements and development of meaningful e-learning applications based on the reusable Learning Objects (LO).

We have been discussing several projects at LEGE-WG national node level, all in different subject domains, as potential e-learning applications-candidates to be designed and deployed as e-learning services on the Grid. Among them the Web based TestTool(TT) developed at KTU for assessment of skills and understanding, which has been piloted and which has been widely used in real educational settings in the Informatics faculty [6]. This paper is a continuation of our work in shaping e-learning applications for the Web service oriented infrastructure [7]. It explores the connection between an established form of testing and the new possibilities for organizing online learning experiences as Web services based on distributed learning objects.

\section{TESTTOOL ARCHITECTURE AND DOMAIN MODEL}

The conventional version of TT is an autonomous, though specific learning management system aimed at providing testing-assessment and self-evaluation (training) learning services. As shown in the system deployment diagram in Fig. 1, TT consists of three packages of cohesive functionality - AuthorTool, StudentTool, AdministratorTool under the control of TestToolServer. Conceptually, the Author and the Administrator tools serve for preparation and building tests to be taken by the students accessing testing materials via StudentTool interface. The Apache Web server is a kernel of TT server along with the database application server and a set of scripts implement resource sharing model typical to the testing domain.

Respectively, we distinguish three categories of TT users as roles: the author, the administrator and the learner. The author's role is testing content creation, i.e. creation of questions. The administrator's wide-angle role, in general, is a creation and deployment of tests. This role may be performed by instructors, tutors and even authors depending on TT exploitation organizational factors. Students may only take tests in exam-assessment or learning mode, actually for them assessment the Web service is to be built.

What kind of learning objects "circulate" in a testing domain? The domain is comprised of three conceptual LOs question, test and course. The smallest one is a question, the largest is a course. The question is a logical entity 
consisting of content and a mechanism to accept and evaluate the answer provided by the learner [6]. For this particular purpose the correct answer is always associated with the given question.

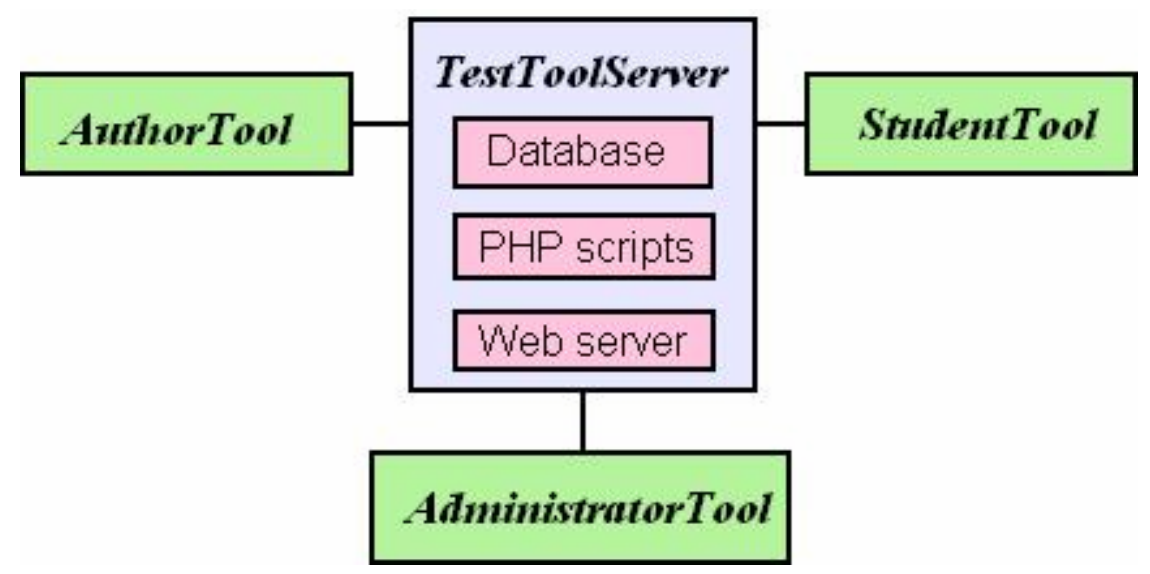

FIGURE 1: TestTool structure

Among traditional forms of test questions, TT implements a specific form of graphical construct type of a question, which enables the instruction designers to simulate task solving situations typical for 'learning by doing' approach. Answering this type of questions, a learner is forced to perform meaningful constructive actions with objects displayed on a graphical panel. Like in the case of Evidence-Centered Design [8] the assessment of skills and understanding is derived from the result of behavioural sequences that learner's interactions with graphical task produce.

A certain number of questions being aggregated into a sequence make up a larger LO - test. The test is a logical entity which is to be made accessible to the learners either in self-testing or in the final assessment (exam) mode. This work in the conventional TT system may be done by the instructor, tutor or administrator. The user browses a set of questions which are related to a given chapter of the subject, looks at their description (metadata) and selects certain ones to be included into the test sequence. The test construct is a temporal unit built and destined to be used within some time period by a group of learners. Typically, this process is called LO packaging. According to LO standards it builds an archive of learning objects with a header attached, which is called manifest [9]. The archive is targeted to transmit over the network to LMS for deployment. Assembling a set of question objects into a bigger test object aiming to deploy this bigger LO as a web service has a principally different meaning from packaging. We'll discuss it in the following section.

The course is a top granularity LO in the testing domain, but it is not a typical content object to be used by learners. It is rather a collection of tests which belong to one subject-module. The instructor or tutor is usually responsible for granting the students access rights to individual tests. This is anticipated as a learning management function referable to the administrator's package (Fig. 1).

The functionality of any of the three packages comprising TT can be made accessible for users as Web services. The central functionality, however, is considered to be the testing service attributable to the online learning session. Therefore, the student package has been taken to implement as a Web service experimental solution in accordance with Service Oriented Architecture (SOA) standards. The implementation of testing as a Web service within the particular TT domain appeared compelling in consideration of a few factors. Firstly, a question object is too small to be used as a pedagogical unit, the test object as a meaningful unit has to be assembled. The building of a testing Web service using individual learning objects implies a shift from a resource sharing model to a service composition from the 'chunks' of learning material - Learning Objects (LO). Secondly, TT domain has a specific graphical construct question which enables the instructors to model problem solving situations typical to experiential learning. Finally, the conventional TT implementation has already proved to be effective in the educational process and the existence of the system prototype facilitated the development of appropriate Web services for the testing environment and moving toward Grid aware TT version.

\section{THE DESIGN MODEL}

The conventional TestTool implements a resource sharing model and is realized in accordance with the domain model described above, using Java programming environment and applets. The shift from resource sharing to 
service composition when building the Web service oriented architectures is a principal step which affects the programming model used for building Grid applications.

In order to build a testing Web service, the domain learning objects have been mapped to respective software objects and represented as software classes with their signatures specified. The design model, as shown in Fig. 2, slightly extends the domain model introducing hierarchy at the Question level. The Question class may have several Variants, each of them implicitly possessing the structure and behaviour of the Question. Conceptually, this simple inheritance hierarchy models the possibility to address a question and to select randomly one of its variants to be presented to the learner during the testing session. In other words, any Variant is treated as an instance of the Question class.

Question variants are created by the authors making use of a special TT graphical editor. Physically, the core of any Question is XML data file wrapped into the structure along with the answer evaluation mechanism and built up in this way as an executable software object which is capable of 'driving behind' the Web service. On the other hand, a Question as a LO may be supplied with associated standard metadata, which evidently should be useful in the case of selecting the object as an item when composing the test service. In terms of LO metadata, a Question is not an expositive content object, but an active object which should be accessed and invoked during the testing session.

Having created a number of questions, which belong to the given subject, the instructor or tutor may want to build a Test - a compound LO to be taken by students during self-training or the exam session. This point is a critical choice from two -a composition of a bigger compound LO on which testing Web service should be grounded, or the composition of the Web service as a collection (arbitrary list) of individual LOs. The principal difference between those two is that the compound LO wraps individual LOs and hides their interfaces, whilst the list assumes 'poor coupling' retaining individual LO's interfaces. This experimental testing Web service is realized according to the first approach - the test is the instance of Test design class, interfaces of which are shown in Fig. 2.

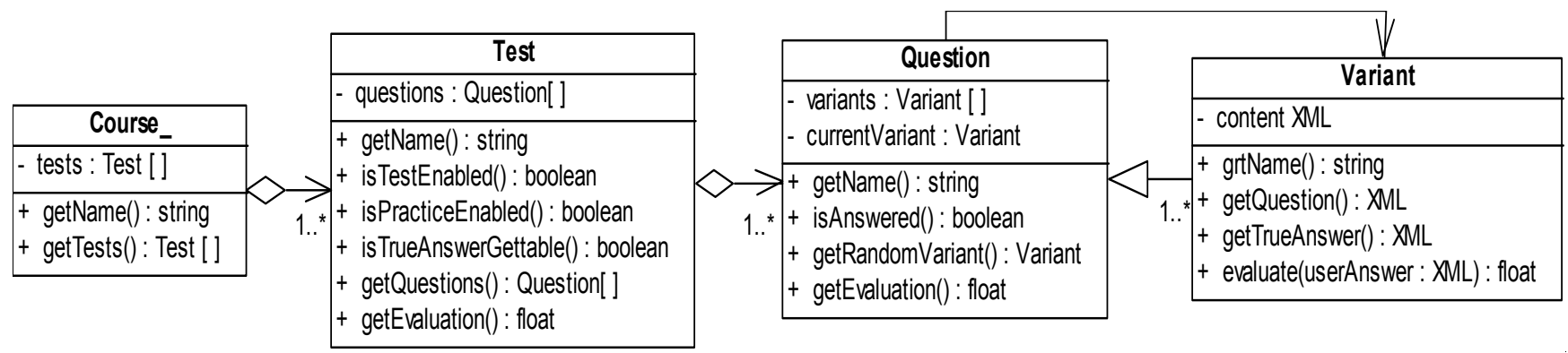

FIGURE 2: Design class diagram

The Course is a top level design object. It models a set of tests, each test belonging to the same teaching module or subject. In the context of the TT execution environment, the Course object has a known responsibility such as information regarding the existing tests and their availability. Obviously, the integration of tests into the top level granularity Course object is a function for the TT administrator or tutor. This is a kind of a management function, which may be included into the administrator's Web service later.

The Java Web Service Developer Pack (JWSDP) programming tools have been used to build the experimental assessment and testing Web service for learners. Obviously, the JWSDP as a fundamental technology platform to build Web services provides a full set of instruments to implement a manageable, secure, robust web service, but our interest was focused only on the web service composition along with the acquirement of means and software tools aimed at building interactive e-learning services. The building process may mistakenly appear quite simple and straightforward because the main job in this case was done by creating the compound Test design class with clearly defined interfaces. In particular, a subset of JAX-RPC has been used to build the WSDL file for the testing service while the service linkage with the conventional TT environment was ensured by a few special purpose Java programs. In terms of time-line characteristics, the Web service based testing has a longer response time in comparison with resource sharing, but the difference is not significant.

\section{DISCUSSION}

The learning Web service implementation when dealing with specific to testing environment learning objects has been described with the focus on building the Web service and it's WSDL. In more general terms, the authors 
create individual LOs and supply metadata to make them reusable. A selection of objects and building the learning Web service which utilizes them is both a pedagogical and technological issue. The pedagogical issue is considered as an instructional design when the instructor looks at LO's metadata and selects the objects which serve him best to achieve the learning objective. The technological issue is to build a service out of LOs selected. Taking into account that among learning objects may be not only expositive content objects but also active not downloadable components with various interfaces makes a service building not so trivial.

In this paper we analysed only the technological issues. From the perspective of the Web service composition, the advantageous feature of the TT design is that homogeneous LOs - Question and Test objects - are being used. Every question instance, though it represents a different question type, belongs to the Question class with unambiguously defined interface. Equally, any test instance belongs to the Test class. This fact allows us to build a WSDL for the test class and to have it deployed for every new Test instance created. In this experimental TT version assembling the questions into a compound test LO is ensured by a few special purpose Java classes.

Meanwhile, in a general case of e-learning service composition from reusable learning objects it is more likely, that individual LOs have different interfaces. They are not only homogeneous as in the TT environment. Moreover, it is more likely that the objects are distributed on several hosts. Even in the practical use of TT in real education various e-learning scenarios tend to occur. An example of such the scenario from the Data Structures course was discussed [10], where linear list simulation program example was included.

Simulations are usually centered around the students' activities and are effective in helping them to gain a better understanding of the material. A simulation usually has inputs that should be meaningfully manipulated and fed into the model to see what outputs are produced. Hereby, the simulation LO most probably has a signature which differs from the Question object signature. The need to compose a Web service which involves both Questions and simulation learning objects breaks the homogeneity of interfaces. How should learning Web service be built in cases like this, when various expositive and active objects have to be included according to the given instructional design?

As it has been mentioned earlier [7], the greater role in shaping e-learning service should be given to LO metadata. The requirements for LO metadata in order to appropriately support pedagogic and economic goals when LOs are intended to be used in service-oriented architectures like the Grid were analyzed [11] and some insufficiencies were pointed out in the existing IEEE LOM standard. The software technology requirements for metadata when reusable LOs are being thought of as services are also important. In particular, when LO is a non-downloadable software component, its interfaces could or should be adequately specified in the associated metadata in order to build an automatically appropriate service description containing a full access specification for the component's method invocation. Meantime, the current version of IEEE LOM standard under $5^{\text {th }}$ category "Educational" describes active LO as a content that includes simulations, questionnaires, and exercises, which "prompts the learner for semantically meaningful input or for some other kind of productive action, not necessarily performed within the learning object's framework" [12, p. 24]. Only the location of LO specified by the URL may directly be used when building the Web services. Supporting the opinion that "some major improvements will have to be made concerning the support of delivery of high quality e-Learning LO services" [11] in the meta-tagging of LO, our objectives for further development are to investigate the technological issues of setting a learning Web services from the reusable LO. The experience gained when building testing service contributes to that, and also may be useful for setting and implementing SEES for E_LeGI project.

\section{REFERENCES}

[1] Grid and Web Services Standards to Converge (2004). The Globus Alliance (http://www.globus.org) announcement at http://www.marketwire.com/mw/release_html_b1?release_id=61977

[2] G. Vossen, P. Westerkamp (2003) E-Learning as a Web Service. Proceedings of the Seventh International Database Engineering and application Symposium (IDEAS'03), IEEE Computer Society.

[3] N. Capuano, et al. (2003) How to Use GRID Technology for Building the Next Generation Learning Environments. 2nd International LEGE-WG Workshop on e-Learning and Grid Technologies: a Fundamental Challenge for Europe, Paris, France, 3rd \& 4th March 2003, British Computer Society, Electronic Workshops in Computing (eWiC) http://www.bcs.org/ewic/

[4] R. Barauskas (2003) Education in Finite Element Analysis and Applications in Computational Grid Networks Advanced Learning Technologies and Applications, Proc. of the International Conference, Kaunas Univ. of Technology, 11-12 Sept., Lithuania, p.p. $34-38$. 
[5] Yanlin Zheng, et al. (2003) Using Knowledge Grid to Construct Next Generation E-Learning Proceedings of E-Learn 2003 World Conference on E-Learning in Corporate, Government, Healthcare, and Higher Education, November 7-11, Phoenix, Arizona USA, p.p. 1849-1852.

[6] K. Baniulis, V. Reklaitis (2002) TestTool: Web-based Testing, Assessment, Learning Informatics in Education, Institute of Mathematics and Informatics, Lithuanian Academy of Sciences, Vilnius, vol. 1, p.p. 17-30.

[7] V. Reklaitis, K. Baniulis, T. Okamoto (2003) Shaping e-Learning Applications for a Service Oriented GriD. 2nd International LEGE-WG Workshop on e-Learning and Grid Technologies: a Fundamental Challenge for Europe, Paris, France, 3rd \& 4th March 2003, British Computer Society, Electronic Workshops in Computing (eWiC) http://www.bcs.org/ewic/

[8] M. Bauer, et al. (2003) Evidence-Centered Design to Develop Advanced Simulation-Based Assessment and Training. Proceedings of E-Learn 2003 World Conference on E-Learning in Corporate, Government, Healthcare, and Higher Education, November 7-11, Phoenix, Arizona USA, p.p. 1495 - 1502.

[9] IMS Content Packaging Information Model. (2003) IMS Global Learning Consortium. Version 1.1.3 Final Specification, http://www.imsglobal.org/content/packaging/cpv1p1p3/imscp_infov1p1p3.html

[10] K. Baniulis, at al (2003) Case study of virtual organization learning and knowledge testing environments. 3rd International LeGE-WG Workshop: GRID infrastructure to support future technology enhanced Learning, Berlin. 3 Dec., http://ewic.bcs.org/conferences/2003/3rdlege/index.htm

[11] K. Wulf (2003) Reusability of e-Learning Objects in the context of Learning Grids. 3rd International LeGEWG Workshop: GRID Infrastructure to support future technology enhanced Learning, Berlin. 3 Dec., http://ewic.bcs.org/conferences/2003/3rdlege/index.htm

[12] IEEE LOM. (2002) Draft Standard for Learning Object Metadata, http://Itsc.ieee.org/wg12/files/LOM_1484_12_1_v1_Final_Draft.pdf 